Chirurg 2019 90 (Suppl 2):S60 https://doi.org/10.1007/s00104-019-0908-2 Online publiziert: 13. Februar 2019

(c) Springer Medizin Verlag $\mathrm{GmbH}$, ein Teil von Springer Nature 2019

\section{Originalpublikation}

Gelli M, Allard MA, Farges O, Paugam-Burtz C, Mabrut JY, Regimbeau JM, Vibert E,

Boleslawski $E$, on behalf of the Association de Chirurgie Hepato-Biliaire et de

Transplantation Hépatique (ACHBT)-French Hepatectomy Study Group. Use of aspirin and bleeding-related complications after hepatic resection. Br J Surg 105(4):429-438

Einleitung. Dauerhafte Thrombozytenaggregationshemmung (TAH) mittels Acetylsalicylsäure (ASS) oder P2Y12-Inhibitoren (Clopidogrel oder Ticlopidin) wird vielfach zur Prävention kardiovaskulärer Erkrankungen verwendet. Bei kardiochirurgischen Eingriffen ist die perioperative Therapie mit Aspirin nicht mit einem erhöhten Komplikationsrisiko verbunden. Der Einfluss von Aspirin auf das postoperative Outcome und auf blutungsassoziierte Komplikationen nach Leberchirurgie ist bisher jedoch nicht in Studien untersucht. Dies ist daher Ziel der vorliegenden Arbeit.

Methodik. Alle Patienten, die zwischen April 2012 und Juli 2015 in 5 französischen hepatobiliären Zentren eine kurative, elektive Leberteilresektion erhielten, wurden in die Studie eingeschlossen. TAH wurde als tägliche Einnahme von ASS (75-150 mg/d) und/ oder P2Y15-Inhibitoren (Clopidogrel $75 \mathrm{mg} / \mathrm{d}$ ) definiert. Entsprechend der Unterbrechung bzw. Fortführung der

Dieser Beitrag wurde erstpubliziert in Der Chirurg (2018) 89:929-929. https://doi.org/10. 1007/s00104-018-0721-3

A. Tekbas - F. Rauchfuß - U. Settmacher

Klinik für Allgemein-, Viszeral- und Gefäßchirurgie, Universitätsklinikum Jena, Jena, Deutschland

\title{
Einfluss von Aspirin auf blutungsassoziierte Komplikationen nach Leberchirurgie
}

TAH-Therapie wurden die Patienten in zwei vergleichbare Gruppen mit dem primären Endpunkt der schweren Blutung nach Dindo-Clavien Grad III-IV aufgeteilt. Die Beobachtungszeit betrug 90 Tage postoperativ. Zur Vermeidung möglicher Bias wurde die PropensityScore-Matching(PSM)-Methode angewendet.

Ergebnisse. Nach Anwendung der PSMMethode enthielten sowohl die TAHGruppe als auch die Kontrollgruppe (K) 108 Patienten. Es konnten keine signifikanten Unterschiede im frühen postoperativen Outcome gezeigt werden. Eine höhergradige Blutung konnte bei 7 Patienten (6,5\%) der TAH-Gruppe und $6(5,6 \%)$ der K-Gruppe gesehen werden (Odds Ratio (OR) 1,18, $95 \%$ CI 0,38-3,62). 25 Patienten (23,1\%) der TAH-Gruppe versus 15 (13,9\%) der K-Gruppe entwickelten eine höhergradige (mind. Dindo-Clavien Grad IIIb) postoperative Komplikation (OR 1,82, 0,92-3,79). Hierbei wurden 6 Fälle mit Leberversagen in der TAH-Gruppe und 4 in der K-Gruppe beobachtet. In beiden Gruppen erfolgte bei 2 Patienten eine Intervention aufgrund einer intraabdominalen Flüssigkeitskollektion. Eine Galleleckage trat lediglich bei 5 Patienten in der TAH-Gruppe auf. Kardiovaskuläre, pulmonale oder infektiöse Komplikationen gab es bei 8 Patienten in der TAH-Gruppe und 7 in der K-Gruppe. In der TAH-Gruppe verstarben 6 (5,6\%) Patienten, hingegen $5(4,6 \%)$ in der K-Gruppe.
Fazit. Die vorgestellte Studie greift die kontroverse Diskussion um die perioperative Unterbrechung bzw. Fortführung der TAH-Therapie bei Leberteilresektionen auf. Während es für die Herzchirurgie diverse Studien gibt, die zu gegensätzlichen Schlussfolgerungen kommen, ist dieses Feld für die hepatobiliäre Chirurgie noch nicht untersucht. Die Autoren kommen zu der Schlussfolgerung, dass die perioperative ASS-Therapie nicht signifikant mit einem höheren Blutungsrisiko assoziiert ist. Allerdings wird die PSM-Methode angewendet, um mögliche Kofaktoren, die die Vergleichbarkeit der blutungsassoziierten Komplikationen beeinflussen könnten, auszuschalten. Ohne die Methode beträgt die postoperative Blutungsrate $11 \%$ in der TAH-Gruppe, verglichen mit 5,3\% in der K-Gruppe. Es wird deutlich, dass weitere Studien notwendig sind, um eine allgemeingültige Empfehlung zur Verwendung von TAH in der Leberchirurgie abgeben zu können.

\section{Korrespondenzadresse}

\section{Dr. med. A. Tekbas}

Klinik für Allgemein-, Viszeral- und Gefäßchirurgie, Universitätsklinikum Jena Am Klinikum 1, 07747 Jena, Deutschland Aysun.Tekbas@med.uni-jena.de

Interessenkonflikt. A. Tekbas, F. Rauchfuß und U. Settmacher geben an, dass kein Interessenkonflikt besteht. 